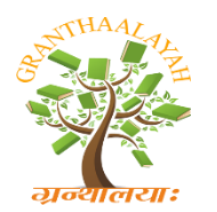

Science

\title{
COMPATIBILITY OF BIOMAGNETIC PROFILES FOUND IN LIVING MATTER BY CROSS SPECIES DEMONSTRATION
}

\author{
Abraham A Embi Bs *1 \\ ${ }^{* 1}$ Independent Citizen Scientist, 13442 SW 102 Lane Miami, Florida USA 33186
}

\begin{abstract}
Biomagnetic research is the study of living entities (plants or animals) weak magnetic emissions. The purpose of this manuscript is to present a historical review and evolution of techniques introduced with the purpose of demonstrating bioelectromagnetic emissions of living tissue; as well as its potential uses in medical research. This will be accomplished by revisiting prior research papers. The hair follicle has been described as a miniorgan, having cellular divisions, thus metabolism. As previously described "metabolism entails electron transfers in both plants (photosynthesis and respiration) and animals (cellular respiration) involving movement of electrons from donor to acceptor along the electron transfer chain thus inducing a current within each cell and from cell to cell. According to Faraday's Law and the Hall Effect, these currents induce electromagnetic fields (EMFs) perpendicular and horizontal, respectively, to the plane of the living tissues". Originally the recordings entailed use of complex magnetic shielding and instrumentation. Recently, two tabletops microscopy techniques were introduced, the first using a Prussian Blue Technique mixed with fine iron particles (2000 nanometers in diameter), the second utilizes a three-tier methodology, where cross species magnetic profiles compatibilities are recorded. The utility and medical applications of the two tabletop techniques are discussed
\end{abstract}

Keywords: Human Hair Follicle; Magnetic Profiles; Linear Lorentz Forces; Cyclotron Resonance; Cross Species Biomagnetism.

Cite This Article: Abraham A Embi Bs. (2018). "COMPATIBILITY OF BIOMAGNETIC PROFILES FOUND IN LIVING MATTER BY CROSS SPECIES DEMONSTRATION." International Journal of Research - Granthaalayah, 6(8), 84-92. https://doi.org/10.29121/granthaalayah.v6.i8.2018.1264.

\section{Introduction}

\section{Brief History of Human Biomagnetic Research The Magnetic Shielding Era}

The field of Biomagnetism is the study of living entities low-level (weak) magnetic emissions. The challenge encountered by early researchers was how to shield the very weak magnetic fields emitted by the human body from outside interference. This was accomplished by utilizing heavy magnetic shielding and a magnetic gradiometer. 
Records show that in the early 1960' the first report of Electromagnetic Fields (EMFs) measurements were done from the human body (1). Progress continued by recording EMFs from the brain, heart and hair follicles $(2,3,4)$. This pioneer work utilized magnetic shielding and sophisticated sensors. This manuscript re-introduces a tabletop microscopy technique that enables the user to easily display individual or paired magnetic profiles amongst living entities in an ecosystem.

\section{The Prussian Blue Stain/iron Era}

A major breakthrough in biomagnetic research occurred in 2015 by the introduction of a tabletop optical microscopy technique that allows for the recording of weak electromagnetic signals from crystals accretion of an evaporated solution containing Prussian Blue Stain (PBS) and fine iron particles (5). The PBS/iron technique relies in the paramagnetic property (attracted to a magnetic field) of iron imbedded in PBS crystals. This approach resulted in numerous publications by this author and others. For example, a paper in particular documented for the first time the inherent property of electromagnetic energy emitted by the hair follicle (6), EMFs penetrating through glass barriers (7), paired (fronted) hair follicles inhibiting diamagnetic crystals formation (8). Additionally, in the field of cross-species biomagnetism, similarities were documented between mosquitoes and humans hairs (9). Further research also showed insect pollinators (ants) and flowering plants parts (leaves edges, pistils) also attracting ferricyanides crystals as a reflection of biomagnetic attraction. This led to a Pollination-Cross Species Biomagnetic Hypothesis (10). Since biomagnetic fields travel through glass, it was also theorized that it could travel through air; therefore papers were published where in vivo hair follicles electromagnetic images were recorded on the skin surface through glass barriers $(11,12)$. A recent paper seems to validate the prior research herein presented, why? Because for the first time by using the crystals accretion approach, the vertical Biomagnetic Field Reach (maximum distance EMFs traveled) of the human hair follicle was measured to be approximately $3 \mathrm{~mm}$. Also introduced is a new paradigm; namely, The Pulsating Nature of Biomagnetism (13).

\section{The Beginnings of the Magnetic Profiles Era}

While observing hair follicles immersed in water drops, on occasions and while the videomicroscope depth of field was being changed, some spatially related concentric and semicircles lines were displayed. The images were sent for review to a renowned physicist and to my surprise, they were interpreted as Lorentz Forces and Cyclotron Resonance patterns (13). This led to the first paper demonstrating magnetic profiles patterns on the mosquito proboscis and human blood. These profiles were hypothesized as factor in under the skin blood source location by the female mosquito (14). Please visit Materials and Methods section for further details. http://www.jnsci.org/content/186

\section{Additional Demonstration of Plants and animals Biomagnetic Profiles}

\section{Hair Follices Biomagnetic Profiles}

Since the hair follicle has been described as a miniorgan, having cellular divisions and metabolism, it was chosen for the display of magnetic profiles. Utilizing the technique previously described, a series of experiments showed through images and video-recordings of some unique findings displayed Figures 1 through 11 as follows: 


\section{Fronted Human Scalp Follicle Profiles}

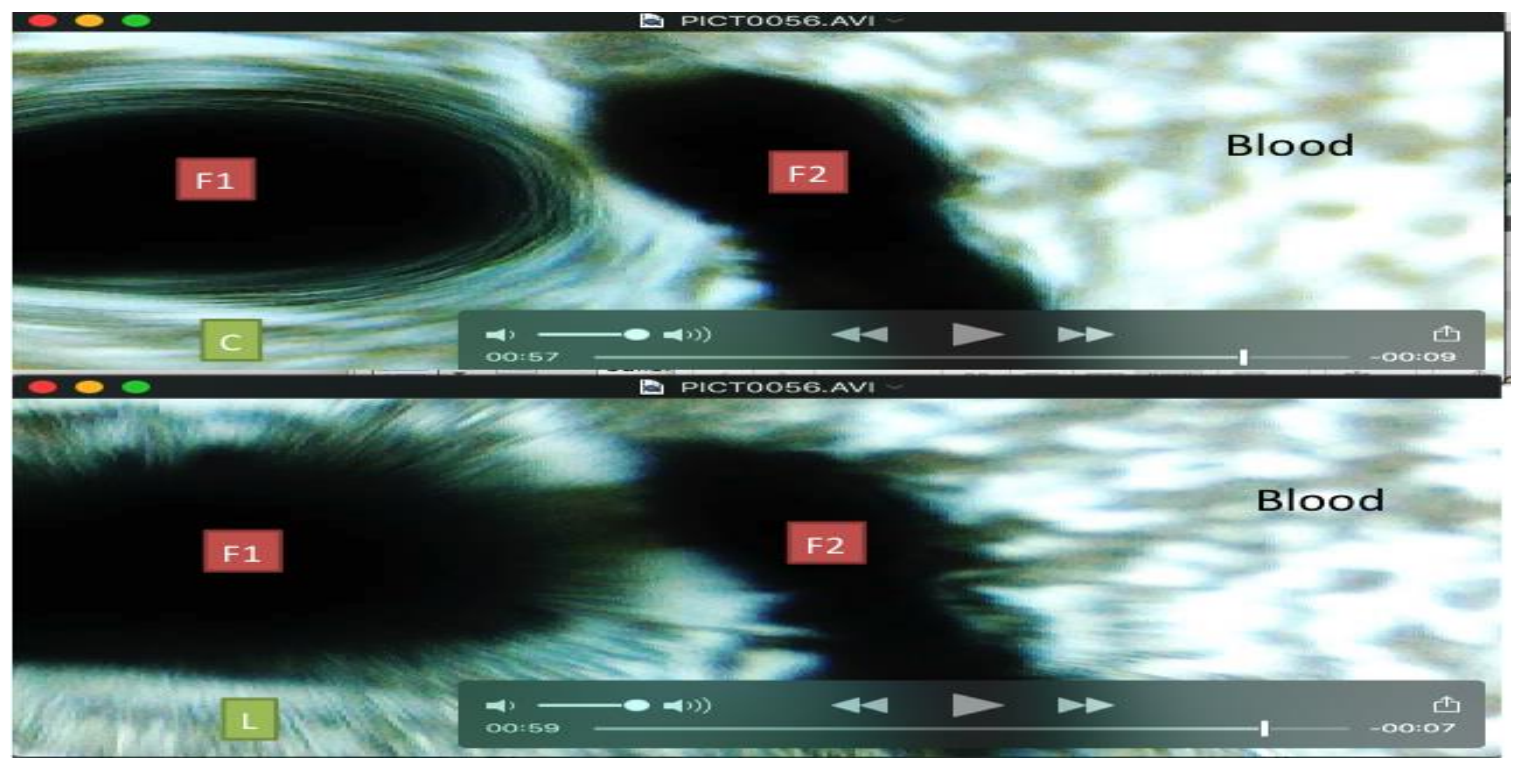

Figure 1: Technique validatiog living tissue individuality. Two hair follicles showing different magnetic profiles. Top slide: F1 (Follicle 1) shows circumferential lines as a product of cyclotron resonance. F2 (Follicle 2) did not express a magnetic profile. Bottom slide: F1 (Follicle 1) again showing magnetic profile express as Lorentz Forces. F2 (Follicle 2 did not express magnetic profiles

\section{Hair Follicles Growth Stages}

The hair shaft grows aided by cell divisions in the hair root or follicle. The hair follicle undergoes growing phases such as Anagen (active growth phase), Catagen (resting phase); and telogen (Regression phase). The example below depicts two fronted hair follicles in either Anagen or Catagen, showing compatibles linear Lorentz Forces.

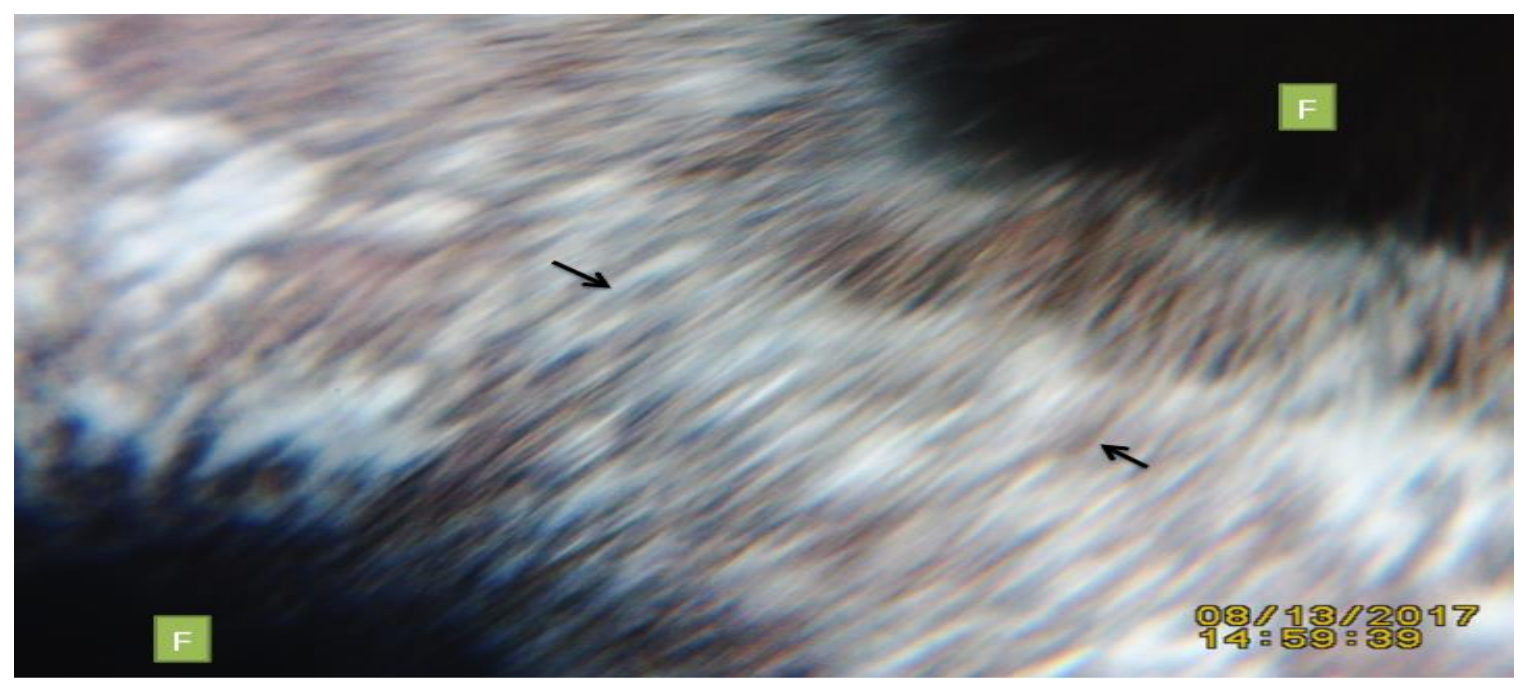

Figure 2. Another example of equivalent size follicles in Anagen (active phase) or Catagen (resting phase)) showing magnetic profile. F= Follicle. Black Arrows= Magnetic Profile lines.

This figure is amplified to show better details of the Linear Lorentz Forces 
Non-living matter (cotton string) void of magnetic profiles

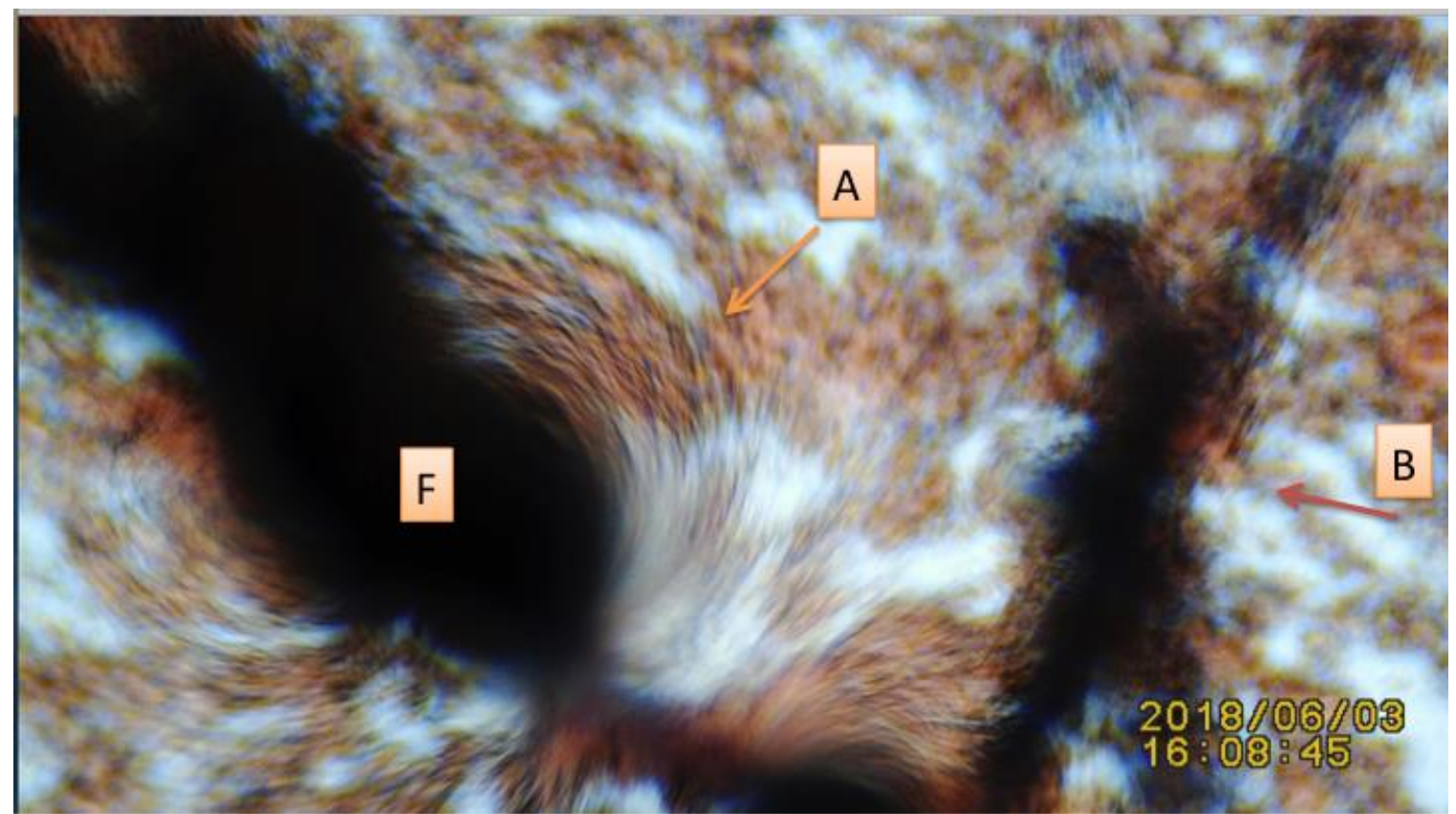

Figure 3: Showing lack of magnetic profiles emitted by an inanimate cotton string. F= Hair follicle $\mathrm{A}=$ Fresh hair in water drop showing magnetic profile $-\mathrm{B}=$ Inanimate object (string) void of magnetic profiles

In this section are examples of fronted living ants magnetic profiles Magnetic Profiles of Fronted Ants

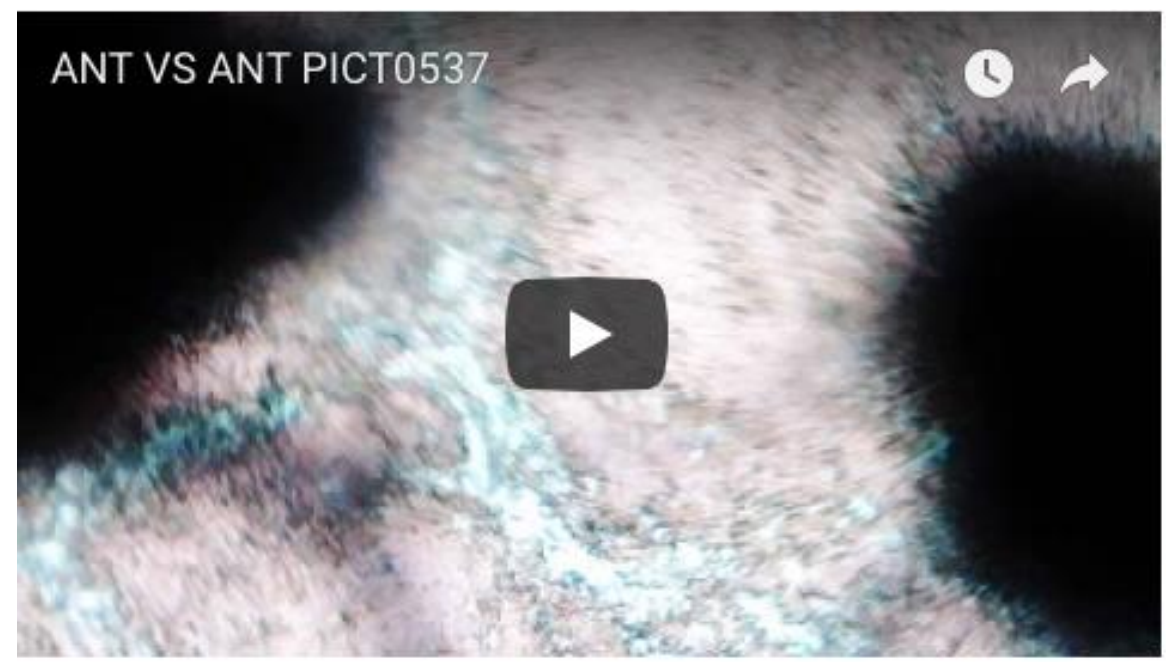

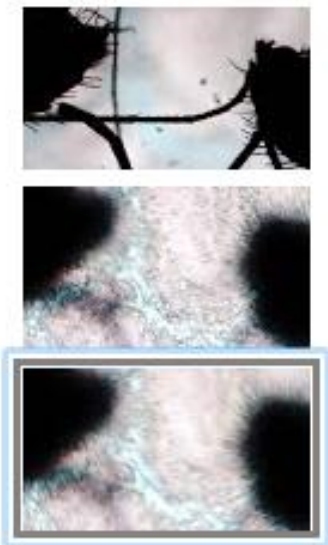

Custom thumbnai

Figure 4: Please visit video-recording for further details: https://youtu.be/WpmDIBAKIRY

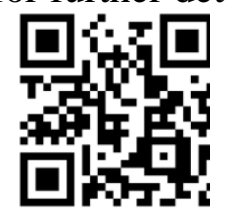




\section{Example of Mosquito body parts magnetic profiles}

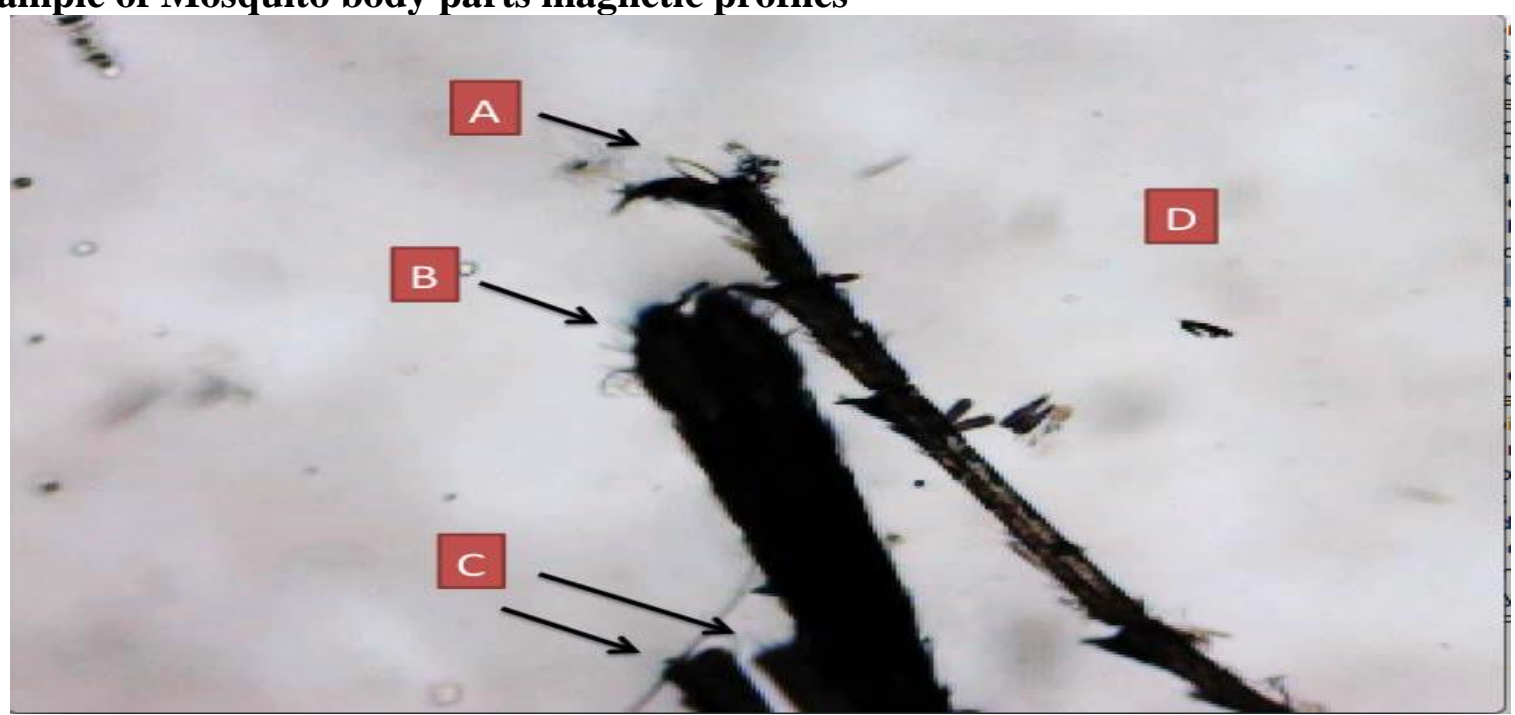

Figure 5: Human blood sandwiched between two $25 \times 75 \times 1 \mathrm{~mm}$ glass slides. On top slide female mosquito parts $\mathrm{A}=$ Distal leg $\mathrm{B}=$ Proboscis $\mathrm{C}=$ Pair of Maxillary Palps $\mathrm{D}=$ Human Blood smear

SDW separated by $1 \mathrm{~mm}$ glass slide. Video microscope images at normal depth of focus.

Cyclotron resonance and Lorentz Forces are seen when changing depth of focus. All three structures express magnetic profiles. Please refer to supplementary video \# 6

https://www.youtube.com/watch?v=Le805XtIxoI

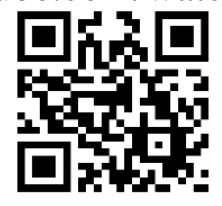

This video recording validates the magnetic profiles technique

Notice the lack of profiles when the mosquito body parts are not influenced by the human blood intrinsic biomagnetism.

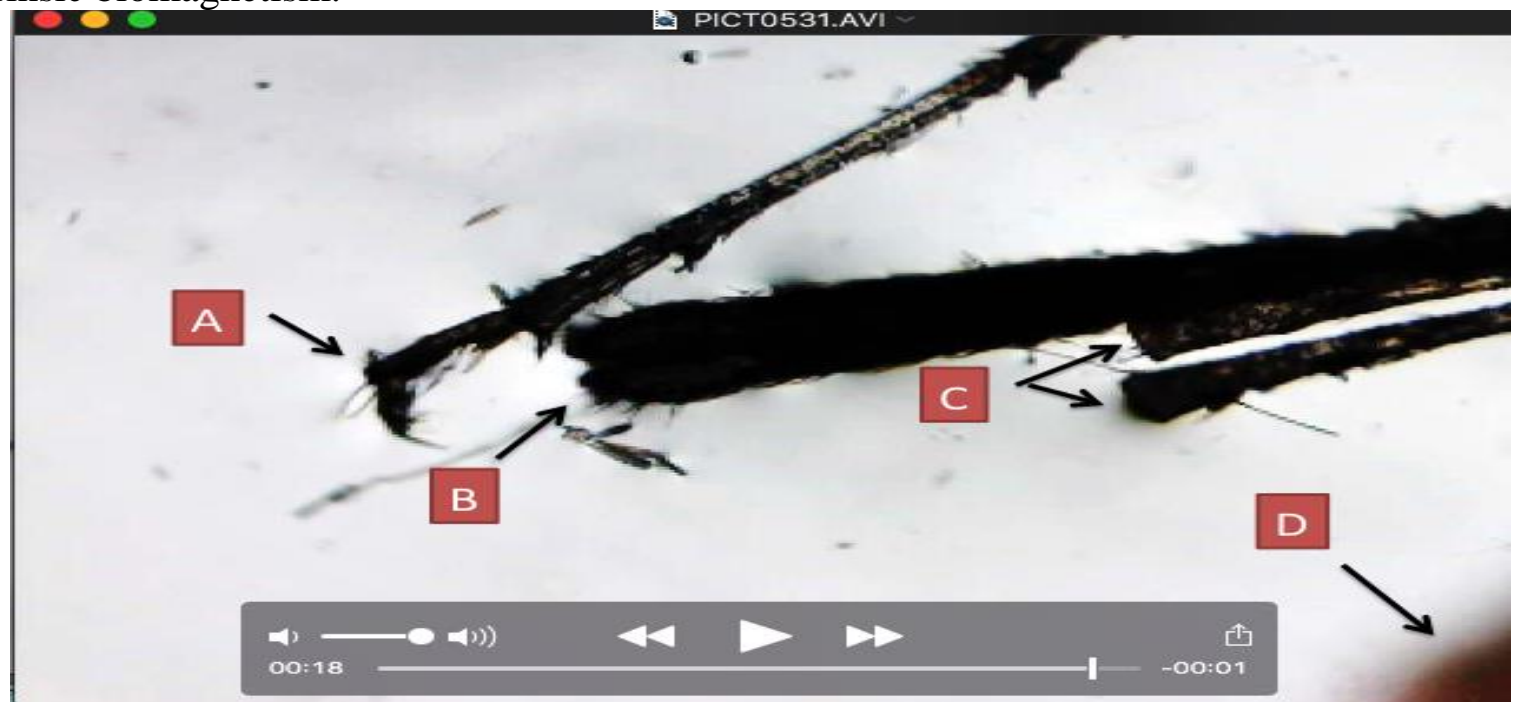

Figure 6. Control experiment. Same female mosquito parts as seen in Figure 7. This time there is zero blood drops sandwiched underneath. The video-recording could not generate magnetic 
profiles as the depth of focus was changed. $\mathrm{A}=\mathrm{Leg} \mathrm{B}=$ Proboscis $\mathrm{C}=$ Pair of Palps $\mathrm{D}=\mathrm{Blood}$ SDW boundary. Please refer to supplementary video \# $7 \mathrm{https} / / /$ youtu.be/CMYSB4hNQYY

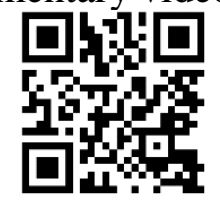

\section{Female mosquito stylet blood seeking mechanism}

Theorized is that the mosquito mouth part entering the skin relies on its own magnetic profile to detect the biomagnetic signal from circulating blood in a capillary.

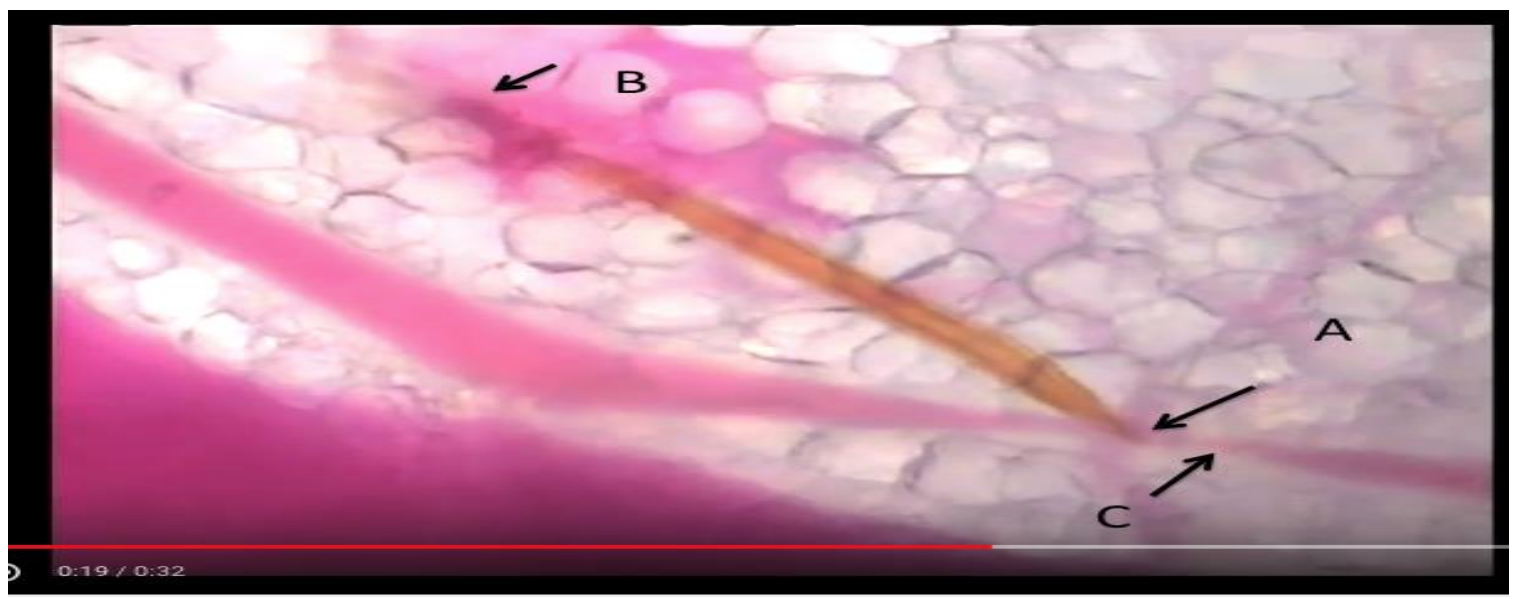

Figure 7: Frame \#019 of video. Mosquito stylet under skin blindly cannulating capillary. $A=$ Proboscis tip $\mathrm{B}=$ Skin entry point $\mathrm{C}=$ Blood now suctioned into proboscis. Courtesy of Valerie Choumet and the Pasteur Institute, Paris. To view complete video please refer to supplementary video-link. https://www.youtube.com/watch?v=6lpidRxVhx4

\section{Demonstration of insect (ant) and plant reproductive organ (stamen) profiles}

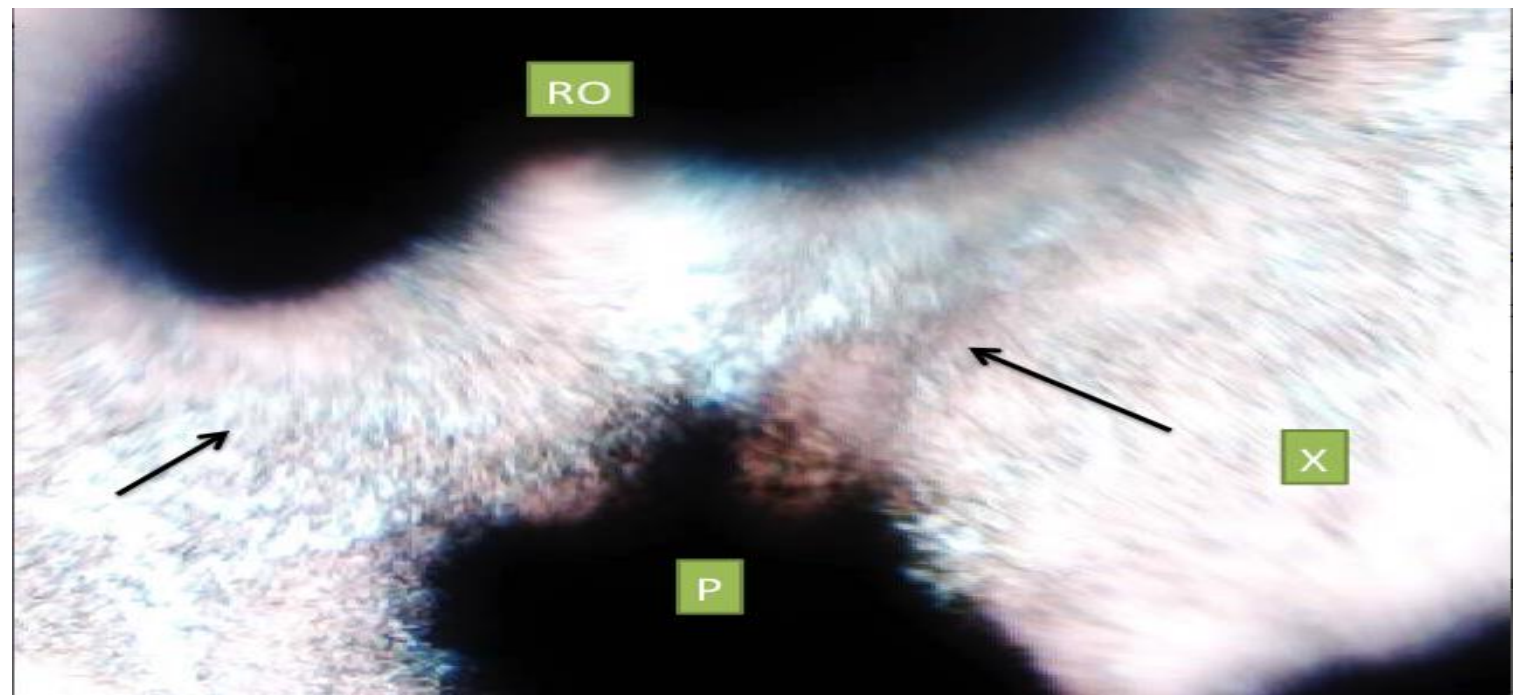

Figure 8: Microphotograph of video frame showing: Black Arrows+ Linear Lorentz Forces RO= Plant reproductive organ $\mathrm{P}=$ Pollinator (Ant) X-Sandwiched blood 


\section{Summary and Conclusions}

A brief summary of the history of Biomagnetic research was presented. The Magnetic Profiles Technique was presented and validated. Two areas of usefulness of the new magnetic technique are:

\section{Medical Applications of the Technique}

Medical applications: This technique could be used as a laboratory tool to aid in the diagnoses of hair loss syndromes, such as Telogen Effluvium (15). Why? Because, for example in Telogen effluvium a reduction in the magnetic profiles would indicate tissue death (16) in the distal follicle (most inner part inside the skin). This phenomenon is clearly shown in the figures below.

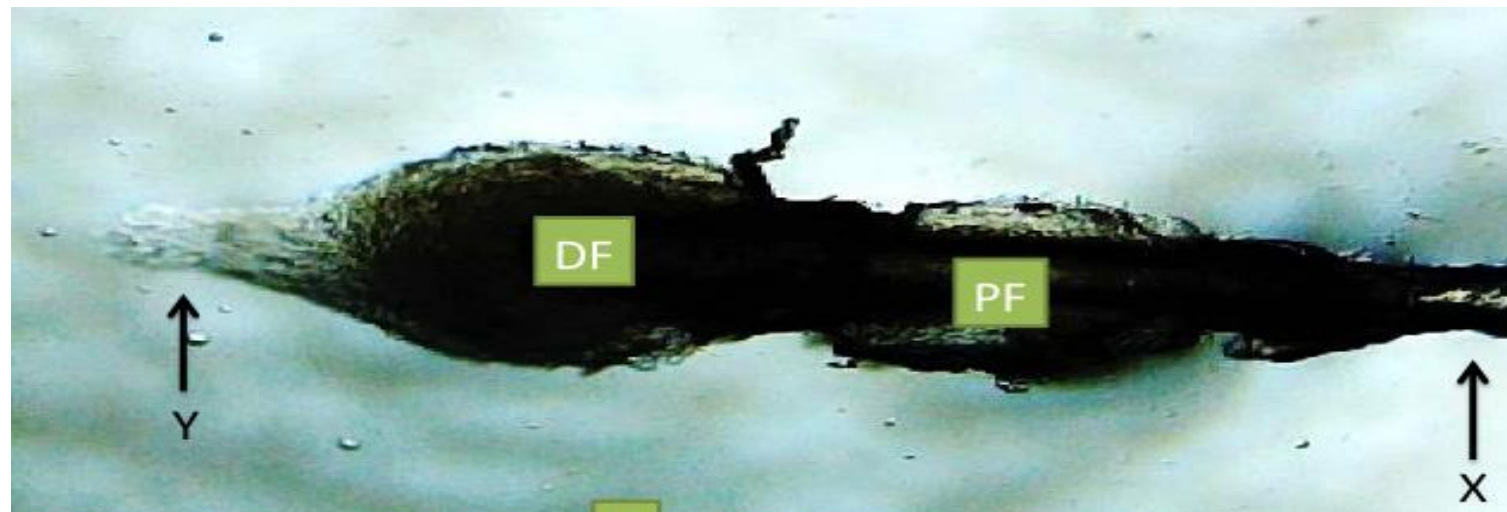

B

Figure 9: Microphotograph of video frame depicting a shrinking human hair follicle in Telogen $\mathrm{X}=$ Hair shaft skin junction $\mathrm{Y}=$ Regressing Epithelium $\mathrm{DF}=$ Distal shrunken follicle $\mathrm{PF}=$ Proximal follicle $\mathrm{B}=$ Sandwiched fresh human blood

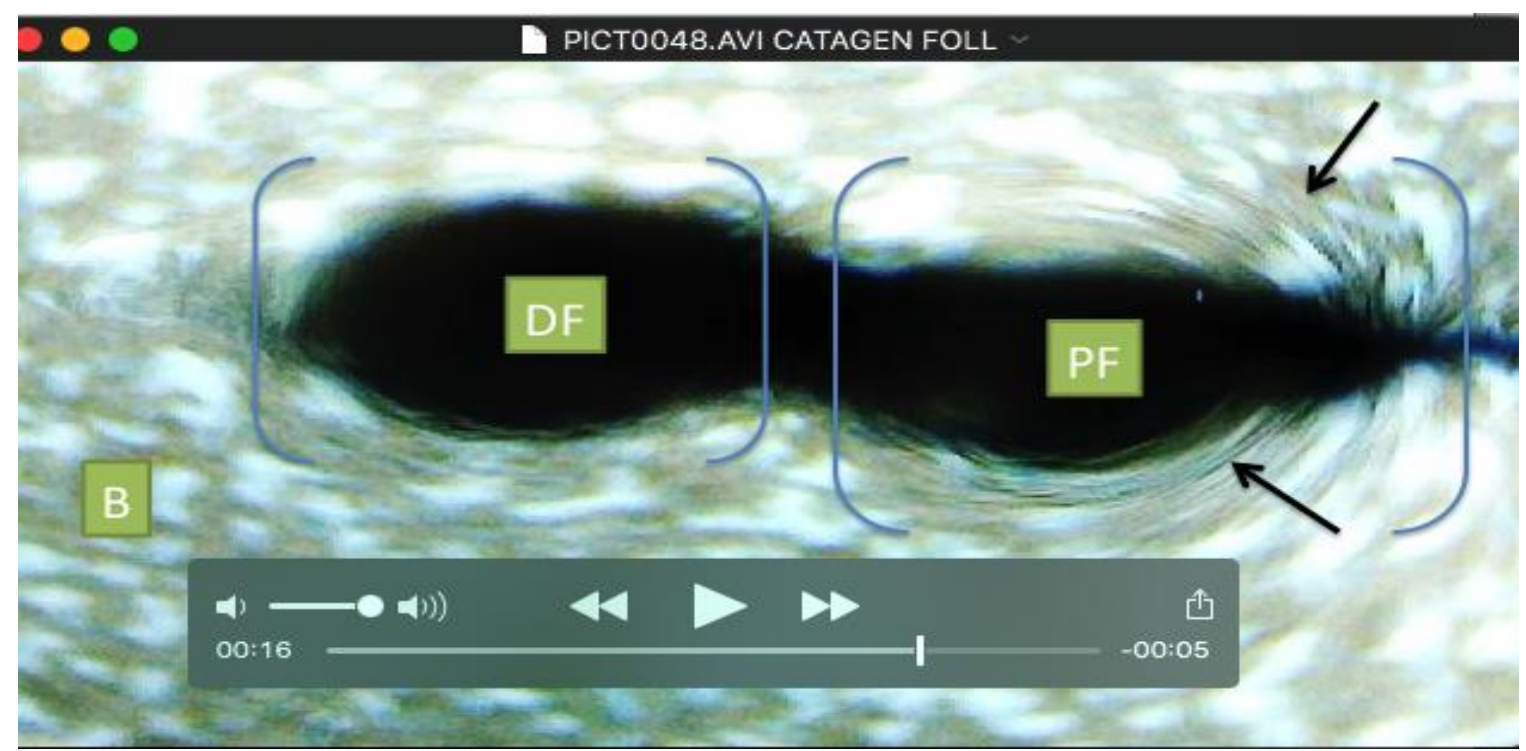

Figure 10: Microphotograph of video frame of hair follicle in Telogen. Same hair follicle in Figure XX showing MPs of a Cyclotron Resonance pattern. $\mathrm{DF}=\mathrm{Distal}$ shrinking follicle $\mathrm{PF}=$ 
Proximal follicle $B=$ Out of focus Sandwiched Human blood smear. Black arrows= Cyclotron Resonance MPs. Notice more noticeable cyclotron resonance profiles in the proximal follicle (PF)

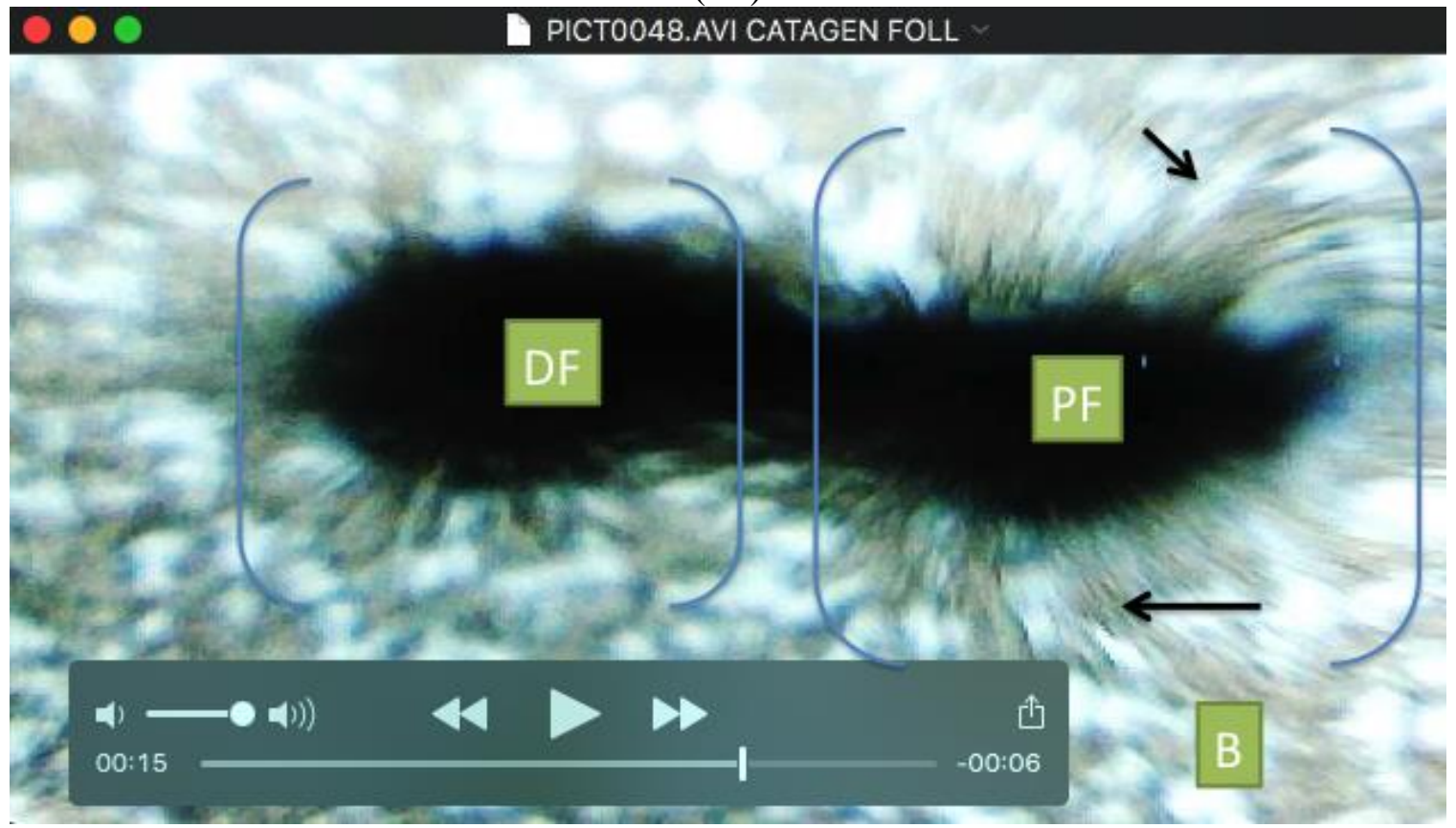

Figure 11: Microphotograph of video frame of hair follicle in Catagen. Same hair follicle in Figure XX showing MPs of Lorentz Forces pattern..DF= Distal shrinking follicle PF= Proximal follicle B= Out of focus Sandwiched Human blood smear. Black arrows= Lorentz Forces profiles. Resonance MPs. Notice more noticeable cyclotron resonance profiles in the proximal follicle (PF).

\section{References}

[1] Baule G.M, McFee R. Detection of the magnetic field of the heart. American Heart Journal 1963;66: 95-96 PBMID: 14045992.

[2] Cohen D. Magnetoencephalography: Detection of the Brain's electrical activity with a superconducting magnetometer. Science 1972;175: 664-666 PMID: 5009769.

[3] Cohen D, Kaufman LA. Magnetic determination of the relationship between the ST segment shift and the injury current produced by coronary occlusion. Circ Res 1975;36: 414-424 PMID: 1111998.

[4] Cohen D, Palti Y, Cuffin BN, Schmid SJ. (1980). Magnetic fields produced by steady currents in the body. Proc. Natl. Acad. Sci. USA; 77(3): 1447-1451.

[5] Scherlag BJ, Sahoo K, Embi AA Novel and Simplified Method for Imaging the Electromagnetic Energy in Plant and Animal Tissue. Journal of Nanoscience and Nanoengineering. Vol 2 No 1, 2016, pp 6-9.

[6] Embi AA, Jacobson JI, Sahoo K, Scherlag BJ. Demonstration of Inherent Electromagnetic Energy Emanating from Isolated Human Hairs. Journal of Nature and Science. 2015; 1: e55.

[7] Embi, AA, Scherlag BJ. Demonstration of Human Hair Follicle Biomagnetic Penetration Through Glass Barriers International Journal of materials Chemistry and Physics. 2016. Vol. 2, No. 2, 2016, pp. 71-74

[8] Abraham A. Embi, Benjamin J. Scherlag. Demonstration of Biomagnetic Responses of Paired Human Hair Follicles Using Nano-sized Iron Particles Solutions: Inhibition of Diamagnetic 
Crystallization. International Journal of Materials Chemistry and Physics. Vol. 2, No. 2, 2016, pp. 84-87

[9] Embi AA. Similarity in Bioelectromagnetic Fields Emitted by Hairs of the Mosquito Larva (Culex quinquefasciatus) and Humans. J Nat Sci, 2(11): e250, 2016.

[10] Abraham A. Embi1, Benjamin J. Scherlag. Bio-magnetism as a Mechanism Underlying the Processes Involved in Pollination. Agricultural and Biological Sciences Journal. Vol. 2, No. 1, 2016, pp. 10-14

[11] Embi AA, Scherlag BJ. Demonstration of human hair follicle biomagnetic penetration through glass barriers. International Journal of Materials Chemistry and Physics. 2016;2: 71-74

[12] Scherlag BJ, Sahoo K. Electromagnetic imaging of subdermal human hair follicles in vivo. Journal of Nature and Science. 2016;2: e-17

[13] Abraham A. Embi Bs. (2018). "THE HUMAN HAIR FOLLICLE PULSATING BIOMAGNETIC FIELD REACH AS MEASURED BY CRYSTALS ACCRETION.” International Journal of Research - Granthaalayah, 6(7), 290-299. https://doi.org/10.5281/zenodo.1341349.

[14] Embi AA. 2016 Human Blood Magnetic Profiles Interactions: Role in Mosquito Feeding. Journal of Nature and Science (JNSCI), 2(3): e186

[15] Malkud S. Telogen Effluvium: A Review. Journal of Clinical and Diagnostic Research: JCDR. 2015;9(9): WE01-WE03. doi:10.7860/JCDR/2015/15219.6492.

[16] Abraham A Embi Bs. (2018). INTRODUCING ANTIOXIDANTS AS ESSENTIAL FOR THE MAINTENANCE OF TISSUE LIFE AS DEMONSTRATED IN HUMAN HAIR FOLLICLES.” International Journal of Research - Granthaalayah, 6(7), 263- 271. https://doi.org/10.5281/zenodo.1341336

\footnotetext{
*Corresponding author.

E-mail address: embi21@ att.net
} 